\title{
ESTUDO SISTEMĀTICO DA FAMÍLIA CICHLIDAE NA REGIÃO CENTRAL DO RIO GRANDE DO SUL. I GENEROS: Aequidens, Cichlaurus e Geophagus
}

Maria Lacy Cezimbra Weis e Ilca Marion Knewitz Bossemeyer Departamento de Biologia. Centro de Ciências Naturais e Exatas. UFSM. Santa Maria, RS.

Horst Oscar Lippold

Departamento de Zootecnia. Centro de Ciēncias Rurais. UFSM. Santa Maria, RS.

\section{RESUMO}

Entre os exemplares da familia Cichlidae, coletados na re gião central do Rio Grande do Sul, foram estudados inicialmente os gêneros representados pelas formas orbiculares: Aequidens, Cichlaurus e Geophagus. Destes gêneros, encontrou-se 4 espécies que ocorreram em proporções bastante variadas: Aequidens portalegrensis, Cichlaums facetus, Geophagus brasiliensis e Geophagus gymnogenys.

\section{SUMMARY}

WEIS, M.L.C.; BOSSEMEYER, I.M.K. and LIPPOLD, H.O., 1981. Systematic study of Cichlidae in the central region of Rio Grande do Su1, Brazi1. I Genera: Aequidens, Cichlaurus and Geophagus. Ciência e Natura (3):65-74.

Among the specimens of the Cichlidae family collected in the central region of the State of Rio Grande do Sul (Brazil), the initial studies were done in genera represented by orbicular forms, which comprise: Aequidens, Cichzaurus and Geophagus. Of these genera, four species were found in different proportions: Aequidens portale grensis, Cichzaurus facetus, Geophagus brasiziensis and Geophagus gymnogenys.

INTRODUÇÃO

Com o objetivo de contribuir para o levantamento da ictio fauna de água doce do Rio Grande do Sul, escassamente conhecida, 0 Núcleo de Ictiologia do Departamento de Biologia da Universidade Fe deral de Santa Maria estabeleceu como sua linha de trabalho o estu do da biologia e sistemática dos peixes da região central deste es tado. Pretende-se, desta maneira, fornecer alguns subsidios que faci litem as pesquisas, básicas ou aplicadas, que se queira realizar no campo da biologia pesqueira.

Consultada a bibliografia disponĩvel sobre a famỉlia Cichli dae, tema do presente estudo, verificou-se que a mesma apresenta lar ga distribuição em toda a Região Neotropical, estando representada 
no estado do Rio Grande do Sul pelos gēneros Aequidens, Cichlaurus, Geophagus, Crenicichla e Batrachops.

Este trabalho, que estuda sobre os gêneros Aequidens, Cichzau rus e Geophagus, marca o inīcio de uma sērie de publicações cuja fí nalidade é preencher, parcialmente, a lacuna existente no éstudo da ictiofauna das āguas interiores desta região.

Os trēs gêneros em questão foram estudados por WALLWITZ(7), apōs coletas realizadas nos municípios gaūchos de Pelotas, Rio Gran de, Camaquã e Santa Vitōria do Palmar.

Registro do gēnero Aequidens, para o Rio Grande do Sul foi feito por RIBEIRO (5) ao descrever a espēcie A. minutus HENSEL, en contrada em um pequeno pântano próximo a Porto Alegre. HENSEL, con siderou Porto Alegre localidade-tipo das espēcies Acara minuta e A. portalegrensis tendo sido esta ūitima anotada por HASEMAN, como Ae quidens portalegrensis para Cacequi e Porto Alegre. FOWLER (4).

Cichlaurus facetus (JENYNS), ūnica espēcie do gēnero com distribuição no Rio Grande do Sul, foi registrada por EIGENMANN, HA SEMAN, REGAN e HENSEL em FOWLER (4). HENSEL considerou Porto Alegre como localidade-tipo de Heros acaroides, sinonímia dada à espécie an teriormente citada. Quanto as duas espécies do gênero Geophagus, por nōs encontradas, temos a confirmação da presença de G. brasiziensis (QUOY \& GAYMARD), no estado, feita por EIGENMANN \& BRAY (3), RIBEI RO (5), MARELLI (em RINGUELET et alii, 6). Esta espécie foi, igual mente, citada em FOWLER (4) como: 10 - G. brasiliensis KNER por HEN SEL para $P$. Alegre; por STEINDACHNER para o Rio Santa Maria (RS) e P. Alegre; por COPE, EIGENMANN, REGAN e IHERING para o Rio Grande do Sul e HASEMAN para Cachoeira e P. Alegre; 20 - G. Zabiatus HENSEL (localidade-tipo: Rio Santa Maria, RS); 30 - G. scymnophilus HENSEL (localidade-tipo: Rio Grande do Sul) 40 - G. pygmaeus HENSEL(locali dade-tipo: P. Alegre, RS).

A espécie G. gymnogenys HENSEL (localidade-tipo: Rio Gran de do Sul), foi também registrada por COPE e REGAN para todo o esta do e por HASEMAN para P. Alegre, Cachoeira, Uruguaiana e Cacequi.Foi descrita como Acara minuta HENSEL (tendo como localidade-tipo P. Ale gre) e como G. camurus COPE (tendo como localidade-tipo o Rio Gran de do Sul). FOWLER (4).

MATERIAL E METODOS

Foi estudado um total de 221 exemplares, assim distribui dos: Aequidens portalegrensis (49); Cichlaurus facetus (1); Geopha gus brasiliensis (164) e G. gymnogenys (7). Este material se encon tra na coleção ictiológica do Núcleo de Ictiologia do Setor de Zoo logia (Departamento de Biologia) da Universidade Federal de Santa Maria. 
Foram realizadas as medidas usuais (expressas em milíme tros), observações e contagens dos caracteres levados em conta para identificação das espēcies da Famīlia CICHLIDAE, os quais se encon tram relacionados, no trabalho, como caracteres específicos. Conven cionou-se às estruturas abaixo, as letras indicadas:

- linha lateral . . . . . . . . . LL

- nadadeira dorsal . . . . . . . . D

- nadadeira anal . . . . . . . . . . A

- nadadeira peitoral . . . . . . . P

REDESCRIÇÃO

FAMILIA Cichlidae Bleeker, 1859

Os ciclídeos constituem uma importante famĩlia de percifor mes que apresenta ampla distribuição geográfica e estā representada na maioria das águas continentais. Os elementos desta famīliamostram uma variação na forma do corpo, que pode ser alongado uu orbicular. Possuem uma linha lateral dividida em dois ramos. Os raios anterio res das nadadeiras dorsal (em maior nūmero) e anal (em número de III ou mais) são transformados em espinhos pungentes. São dotados de es camas ciclōides e ctenóides e de uma escama na axila da nadadeira ven tral. A boca ē anterior, mais ou menos protrātil, com dentes maxila res diferenciados de acordo com os gêneros. (RIBEIRO, 5; DEVINCENZI \& TEAGUE, 2; RINGUELET et alii, 6; BRITSKI, 1 e WALLWITZ, 7 ).

Gênero AEQUIDENS Eigenmann \& Bray, 1894

Tipo: Acara tetramerus Heckel, 1840

Caracteres genéricos: Corpo ablongo e comprimido com escamas ctenói des; perfil dorsal e ventral de contornos semelhantes com boca ante rior e pequena. Frontal sem escamas e provido de moleira. Regiões preopercular e opercular escamosas e dotadas de bordos lisos. Dentes cónicos. Primeiro arco branquial sem 10 bulo dermal e com rastros cur tos de forma tubercular. Nadadeira anal com III espinhos.

Aequidens portaZegrensis (Hense1, 1870)

Eigenmann, 1910

1870 Acara portalegrensis Hensel, Arch. Naturg., 1870: 52. Localida de-tipo: Porto Alegre, RS (Brasil).

1910 Aequidens portalegrensis Eigenmann, Rep. Princeton Univ. Exped. Patagonia III (pt.4): 472 .

Nomes comuns: Cará, acarā e carā-carā

Dimensões: atē $181 \mathrm{~mm}$

No exemplares: 49 


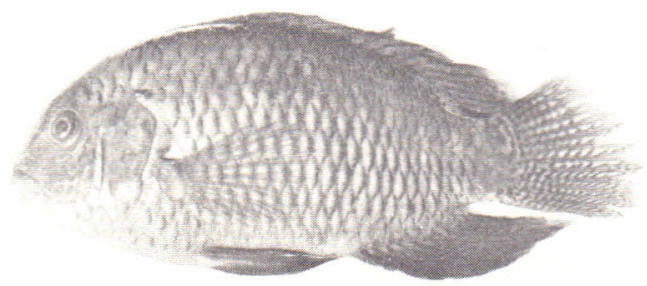

Figura 1. Aequidens portalegrensis (131 mm)

Caracteres especificos:

Escamas ctenōides, grandes, em sērie longitudinal abaixo da linha lateral: 24 a 26; poros $\frac{14-17}{7-10}$; sēries de escamas completas acima da LL ao início da dorsal, entre as duas LL e abaixo da LL ao início da anal: $3+2+5$; raios: D. XIV-XVI + 8-10; A. III + 9-10; P. 14-15. Rastros: $7(1+1+5)$.

Corpo oblongo e forte, comprimido lateralmente, com perfil dorsal e ventral regularmente arqueados. A proporção altura/compri mento é inferior a $1 / 2$. Boca anterior pequena, com premaxilar pouco protrātil e maxilar não exposto distaimente. Dentes cônicos e gran des em uma série externa na mandíbula e maxila, seguida de 3 a 4 f $\underline{j}$ leiras de dentes menores. O ângulo posterior da boca não atinge a vertical que passa pela margem anterior do olho. Três sēries longi tudinais de escamas no preopérculo. 010 arco branquial, sem proces so dermal, é provido de rastros curtos, pouco numerosos e tubercula res. As membranas branquiostegais, na maioria, quase tocam a base da peitoral. Peitoral não atinge a extremidade da ventral; esta, por sua vez, alcança a origem da anal. Dorsal e anal nuas ou com escamas so mente na base e especialmente na região dos maiores raios moles. 0 comprimento do pedūnculo ē de aproximadamente a metade de sua altu ra. Escamas ctenóides moderadas e pequenas, recobrem mais ou menos $50 \%$ da cauda 1 .

Coloração in vivo: Variāvel de acordo com o ambiente. Fundo assumin do tons esverdeadoș, azulados ou amarelados, podendo ser encontra dos exemplares enegrecidos. Apresentam 8 faixas transversais escuras, nem sempre bem visíveis; uma mancha umeral escura (mais ou menos na 3. faixa) e um ocelo no lóbulo superior da caudal. Nadadeiras dorsal, anal e caudal de coloração entre amarelo e marrom,com manchas obli quas e seriadas.

Gênero CICHLAURUS Swaison, 1839

Tipo: Labrus bimaculatus Linnē, 1758

Caracteres genéricos: Corpo orbicular e comprimido, com escamas cte 
nōides e perfil dorsal um pouco mais arqueado que o ventral. Boca anterior e pequena; premaxilar protrātil e maxilar recoberto.Frontal sem escamas, encoberto por pele grossa. Preopérculo escamoso, com bordo saliente e liso; opērculo igualmente escamoso. Ausēncia de pro cesso dērmico na parte superior do 10 arco branquial; rastros com as pecto de pequenos espinhos. Nadadeira anal com mais de III espinhos (de IV a VIII segundo RINGUELET et alii, 6).

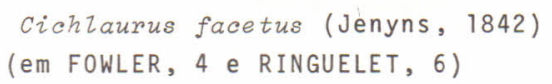

1842 Chromis facetus Jenyns, Zool. Voy. Beagle, pt. 4 Fisches : 104. Localidade-tipo: Maldonado, Uruguay.

1870 Heros jenynsii Steindachner, Sitszb. Akad. wiss. Wien, LX, (pt. 1): p. 292, T. 2. Localidade-tipo: Montevideu.

1874 Acara faceta Steindachner, ibid., LXX (pt. 1): 506. Rio Paranā.

1889 Heros jenynsii: Holmberg, Rev. Soc. Geogr. Argentina VI (cader no 62) 365. Rio de la Plata. Proximidades de Tandil.

1889 Heros facetus Holmberg, ibid.: 365. Rio de la Plata. Riachuelo.

1895 Astronotus (Heros) facetus, Lahille, Rev. Mus. La Plata VI:273 Dique nọ 1 Arroyo del Gato (Buenos Aires).

1903 Heros autochthon, Pellearin, Mem. Soc. Zool. France VII: 235. Buenos Aires.

1905 Cichlasoma facetum Regan, Ann. Mag. Nat. Hist., (Ser. 7), XVI: 70. Buenos Aires, rio Paranā.

1905 Cichlasoma oblongum Regan, ibid.: 72. Argentina.

1911 Cichlasoma facetum Haseman, Ann. Carnegie Mus., VII(3-4):340, t. 57. Uruguayana; Montevideo.

1945 C. obzongum Pozzi, Gaea VII (2): 265. "Rio Uruguay".

1945 C. autochthon: Pozzi, ibid.: 277. "Rio de la Plata ; rio Para nā".

1954 Cichlaurus facetus Fowler, Arq. Zool. São Paulo IX: 292, f. 877.

1957 Cichzasoma autochthon: Ringuelet \& Arämburu, Mrio. Assuntos $\underline{A}$ grārios BS. AS., publ. nọ 119:23. "Paranā-Plata".

1962 Cichzaurus facetum: Ringuelet \& Arāmburu, ibid. (7): 60 .

1962 C. autochthon Ringuelet \& Arämburu, ibid. 60.

1962 C. obzongus Ringuelet \& Arämburu, ibid.: 60.

Nomes comuns: Carā, acarä e carā-carā.

Dimensão: $\quad 163 \mathrm{~mm}$

No exemplares: 1

Caracteres especificos:

Escamas ctenōides, grandes, em sêrie longitudinal abaixo da linha lateral: 25; poros $\frac{14}{1 T}$; sēries de escamas comple 


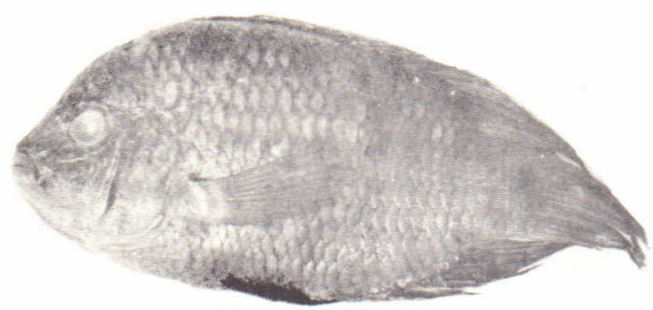

Figura 2. Cichlaurus facetus (163 mm)

tas acima da LL ao inĩcio da dorsal, entre as duas LL e abaixo da LL ao início da ana 1: $3+2+5$; raios D. XVI+10; A. VII+8; P. 14. Rastros: $10(3+1+6)$.

Corpo compacto, rústico, de perfil dorsal mais curvo que o ventral. Boca anterior com premaxilar protrátil em maior ou menor amplitude. Dentes cônicos em uma sērie, seguidos por dentes vilifor mes. Maxilar escassamente exposto em sua porção distal, não alcançan do a vertical que passa pela margem anterior do olho. Mandíbula não proeminente. Narinas mais prōximas da boca do que das ōrbitas. Re gião preopercular provida de quatro sēries longitudinais de escamas ( 3 a 4 de acordo com RIBEIRO, 5 e RINGUELET et alii, 6); região oper cular com escamas grandes. Primeiro arco branquial destituido de pro cesso dermal e provido de pequenos rastros espinhosos. As membranas branquiostegais não atingem a origem das peitorais. As peitorais têm implantação anterior à ventral e não alcançam a extremidade poste rior da mesma; esta ūltima, ultrapassa a origem da anal. A dorsal e a anal possuem escamas atē aproximadamente a metade que vão reduzin do-se gradativamente em nümero. Estas nadadeiras, com seus tērminos de implantações mais ou menos na mesma vertical, ultrapassam em 50\% ou mais a origem da caudal. 0 pedúnculo caudal é curto e alto; sua maior altura é superior ao dobro da distância entre a origem do ūitimo raio da anal ou da dorsal e a base da caudal. Escamas ctenōides, pequenas, reco brem $50 \%$ ou mais da caudal arredondada.

Cozoração in vivo: 0 exemplar estudado, mostrou um fundo esverdeado com 6 faixas transversais mais escuras; este fundo entretanto, se gundo WALLWITZ (7) pode assumir tonalidades variadas. Observou - se ainda duas manchas, sem auréolas, estando uma localizada na metade dos flancos e a outra na base da caudal, ambas ligeiramente acima da linha lateral inferior.

Gēnero GEOPHAGUS Hecke1, 1840

Tipo: Geophagus acutifrons Hecke1, 1840

Caracteres genéricos: Corpo alto, comprimido e robusto; perfil dor 
sal mais arqueado que o ventral. Região frontal nua e levemente côn cava. Boca anterior protrātil e mandíbula não proeminente. 0lho menor que a altura do preorbital que recobre o maxilar. Preopérculo e opér culo escamosos e com bordos posteriores 1isos. Lobo dermal, orlado de rastros tuberculares, no ramo superior do 10 arco branquial. Na dadeira anal com III espinhos.

\section{Geophagus brasiliensis (Quoy \& Gaimard, 1824) \\ Kner, 1869.}

1824 Chromis brasiziensis Quoy \& Gaimard, Voy. Uranie, Zoo1., II:286. Localidade-tipo: Baía do Rio de Janeiro.

1869 Geophagus brasiliensis Kner. Reise Novara, pt. 2: 266, t. 10 f.3 Nomes comuns: Carā, acará, carā-cará e carā-topete.

Dimensões: atē $215 \mathrm{~mm}$

No exemplares: 164

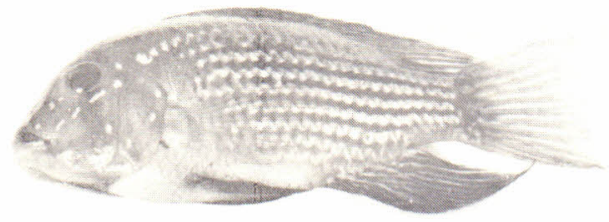

Figura 3. Geophagus brasiziensis (102 mm)

Caracteres especificos:

Escamas ctenōides, grandes, em série longitudinal abaixo da linha latera 1: 27-30; poros $\frac{16-18}{9-13}$; séries de escamas completas acima da LL ao início da dorsal, entre as duas LL e abaixo da LL ao início da anal: $3+2+5$; raios: D. XIV-XVI + 11-14; A. III + 8-9; P. 14. Rastros: 14-16(7+ $1+6),(6+1+8),(6+1+9)$ e $(7+1+8)$.

Corpo mais ou menos ovalado, comprimido, com perfil dorsal levemente mais arqueado que o ventral (principalmente na porção an terior). Altura menor que a metade do comprimento do corpo. Compri mento da cabeça: 3,4 a 3,8 vezes no comprimento total do corpo. Bo ca anterior pequena e com premaxilar protrātil; maxilar não exposto. o ãngulo posterior da boca não atinge a vertical que passa pela mar gem anterior do olho. Escamas no opérculo e nas bochechas ( 3 a 4 sé ries longitudinais). Rastros tuberculares dispostos inclusive no bor do do 1ōbulo dermal. Peitorais curtas, menores que a cabeça, não ul trapassam o começo da nadadeira anal; esta, em alguns exemplares, tinge a metade ou $1 / 3$ da caudal. Ventrais se prolongam até o início da anal. Dorsal e anal geralmente com escamas na base dos raios mo les; as vezes ocorre uma sērie de 2 a 3 escamas somente sobre alguns 
raios. Pedünculo caudal curto, com altura ligeiramente maior que o comprimento. Caudal com bordo arredondado e provido de pequenas es camas que podem cobrir até $1 / 2$ do seu comprimento. Raios posterio res da dorsal e anal e raios externos da caudal se prolongam nos ma chos que podem se apresentar, muitas vezes, providos de giba.

Coloracão in vivo: Corpo pardo azulado ou esverdeado com reflexos me tālicos e 7 faixas transversais escuras; uma mancha também enegrec $\underline{i}$ da no flanco, na altura do tērmino das nadadeiras peitorais. Uma listra escura, nem sempre nítida, atravessa os olhos, desde a nuca até a região inferior do preopérculo. Nadadeiras dorsal, anal e cau dal com manchas escuras e pintas azuis-metālicas; peitorais monocro māticas e ventrais com a margem externa escurecida.

Geophagus gymnogenys Hense1, 1870

Tipo: Geophagus gymnogenys Hense1, Arch. Naturgesch., 1870: 61 Loca lidade-tipo: Rio Grande do Sul.

Nomes comuns: Acarā, carā-carā e carā.

Dimensões: atē $172 \mathrm{~mm}$

No exemplares: 7

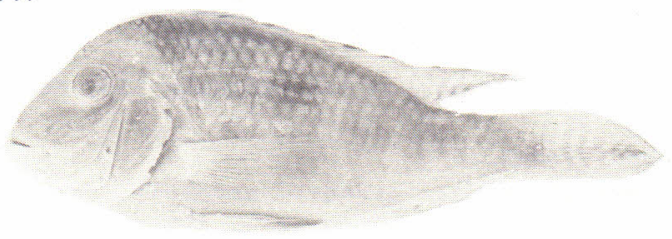

Figura 4. Geophagus gymnogenys (169 mm)

Caracteres especificos:

Escamas ctenōides grandes, em sērie longitudinal, abaixo da linha lateral: 28-29; poros $\frac{17-18}{(8) 10-12}$; sēries de esca mas completas acima da LL ao inicio da dorsal, entre as duas $L L$ e abaixo da $L L$ ao início da anal: $3+2+5$; raios: D. XIII-XIV + 9-11; A. III + 8; P. (13)14. Rastros: $11(5+1$ $+5)$ e $12(6+1+5)$ e $(7+1+4)$.

Corpo piriforme com uma altura pré-dorsal relativamente a centuada e pedúnculo mais 1 ongo que alto ( 1,1 a 1,5 vezes na altura). Comprimento da cabeça: 3,6 a 4,1 vezes no comprimento total. Boca anterior pequena, com premaxilar protrātil e maxilar não exposto. 0 àngulo posterior da boca não atinge a vertical que passa pela margem anterior do olho. Dentes cōnicos no premaxilar e no dentārio. Boche chas destituidas de escamas ou então com escamas moderadamente cte nōides, dispostas em 1 sērie (2 a 3 escamas) sob o olho. Escamas ca ducas no opérculo e subopērculo. Rastros curtos, sendo os do arco 
suderior (4 a 5) localizados no bordo do lobo dermal. Nadadeiras dor sal e anal sem escamas na base. Em exemplares pequenos ambas tocam a caudal e nos maiores atingem $1 / 3$ da mesma. Peitorais longas, iguais ou 1 igeiramente maiores que a cabeça, atingem o começo da anal. Na dadeira caudal truncada e recoberta de escamas atē quase metade do seu comprimento.

Coloração in vivo: Corpo olivāceo com reflexos metālicos e listras transversais escuras, às vezes pouco nîtidas. Uma mancha abaixo da LL mais ou menos na altura do 80 raio da dorsal. Barra escura do in cio da nadadeira dorsal até a linha lateral. Faixa escura atravessan do o olho até a região ventral do preopérculo.

\section{COMENTARIOS E CONCLUSÕES}

Foi possível identificar os gêneros Aequidens, Cichzaurus e Geophagus entre os Ciclídeos examinados quando se comprovou a re lação existente entre as características morfológicas e dados merís ticos por nōs observados e àqueles descritos por EIGENMANN \& BRAY (3), RIBEIRO (5), DEVINCENZI E TEAGUE (2), RINGUELET et alii (6) WALLWITZ (7).

Estes gêneros, quando confrontados com a literatura consul tada, foram separados em 4 espēcies: Aequidens portalegrensis, Cich Zaurus facetus, Geophagus brasiliensis e Geophagus gymnogenys. Des tes exemplares, G. brasiziensis foi o que ocorreu em maior percenta gem $(74,21 \%)$; as demais espēcies apresentaram percentagens correspon dentes a $22,17 \%$ (A. portalegrensis), 3,17\% (G. gymnogenys) e 0,45\% (c. facetus).

Corroborando com a afirmativa de BRITSKI (1) e dos autores anteriormente mencionados, podemos salientar como características mar cantes na separação destes três gêneros: o nūmero de raios espinho sos da nadadeira anal e a presença ou ausência do processo dermal no primeiro arco branquial.

1. Anal com III raios espinhosos ............... 2

la. Anal com IV a VIII raios espinhosos ....... Cichzaurus

2. Processo dermal presente na parte superior do 10 arco

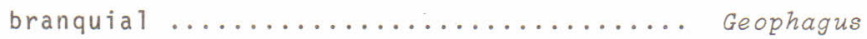

2 a.Processo dermal ausente na parte superior do 10 arco

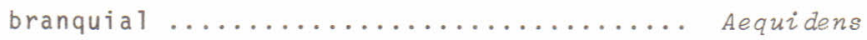

\section{REFERENCIAS BIBLIOGRĀFICAS}

1. BRITSKI, H. Peixes de ägua doce do Estado de São Paulo - Sistemá tica. IN: COMISSÃO INTERESTADUAL DA BACIA PARANÁ - URUGUAI.PO Zuigão e Piscicultura. São Paulo, Faculdade de Saúde Püblica 
da USP e Inst. de Pesca, CPRN, S.A., 1970. 79-108 p.

2. DEVINCENZI, G.J. \& TEAGUE, G.W. Ictiofauna del Rio Uruguay Médio. Anales del Museo de Hist. Nat., Montevideo, 5(4): 1-109, 1942.

3. EIGENMANN, C.H. \& BRAY, W.L. A revision of the americanCichlidae. Annals N.Y. Acad. Sei., VII: 607-24, 1894.

4. FOWLER, H.W. Os peixes de āgua doce do Brasil. Arq. de Zoologia do Est. de S. Paulo, São Paulo, IX: 1-400, 1954.

5. RIBEIRO, A. de M. "Fauna Brasilense" - Peixes(Eleutherobranchios Aspiraphoros) - Physoclisti. Arq. Mus. Nac., Rio de Janeiro, 17: 1-827, 1915.

6. RINGUELET, R.A.; ARAMBURU, R.H. \& ARAMBURU, A.A. Los peces arger tinos de água doce. 1 ed. La Plata, Librart., 1967. 602 p.

7. WALLWITZ, J.C.G. A famīia Cichlidae em Pelotas (RS) com chave para identificação das espēcies. Boletim do IPEMAFLA, Pelotas 2 : 3-21, 1976 .

Recebido em dezembro, 1981; aceito em dezembro, 1981. 